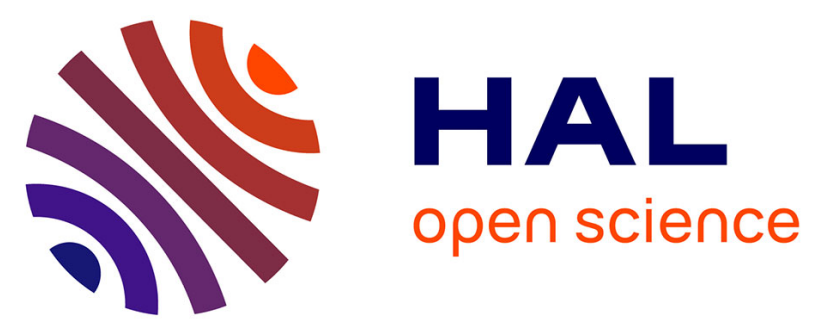

\title{
Characterization of InAs quantum wires on (001)InP: toward the realization of VCSEL structures with a stabilized polarization
}

Jean-Michel Lamy, Christophe Levallois, Abdulhadi Nakkar, Philippe Caroff, Cyril Paranthoen, Rozenn Piron, Alain Le Corre, Abderrahim Ramdane, Slimane Loualiche

\section{To cite this version:}

Jean-Michel Lamy, Christophe Levallois, Abdulhadi Nakkar, Philippe Caroff, Cyril Paranthoen, et al.. Characterization of InAs quantum wires on (001)InP: toward the realization of VCSEL structures with a stabilized polarization. Trends in Nanotechnology 2006, Sep 2006, Grenoble, France. pp.1672-1676, 10.1002/pssa.200675343 . hal-00488399

\section{HAL Id: hal-00488399 https://hal.science/hal-00488399}

Submitted on 1 Jun 2010

HAL is a multi-disciplinary open access archive for the deposit and dissemination of scientific research documents, whether they are published or not. The documents may come from teaching and research institutions in France or abroad, or from public or private research centers.
L'archive ouverte pluridisciplinaire HAL, est destinée au dépôt et à la diffusion de documents scientifiques de niveau recherche, publiés ou non, émanant des établissements d'enseignement et de recherche français ou étrangers, des laboratoires publics ou privés. 


\section{Characterization of InAs quantum wires on (001)InP: toward the realization of VCSEL structures with a stabilized polarization}

J.-M. Lamy ${ }^{*}$, , C. Levallois ${ }^{1}$, A. Nakhar ${ }^{1}$, P. Caroff ${ }^{1}$, C. Paranthoen ${ }^{1}$, R. Piron ${ }^{1}$, A. Le Corre $^{1}$, A. Ramdane ${ }^{2}$, and S. Loualiche ${ }^{1}$

${ }^{1}$ UMR C6082 FOTON - INSA de Rennes, 20 Avenue des Buttes de Coësmes, 35043 Rennes, France

${ }^{2}$ Laboratoire de Photonique et Nanostructures, CNRS UPR20, Route de Nozay, 91460 Marcoussis, France

We propose a new type of long-wavelength vertical cavity surface emitting laser (VCSEL) which consists of quantum wires (QWires) layers of $\operatorname{InAs} / \operatorname{InGaAsP}$ grown on $\operatorname{InP}(001)$ and dielectrics Bragg mirrors, in order to control the in plane polarization of output power. QWires and quantum wells growth are performed by molecular beam epitaxy. QWires present a strong photoluminescence dependence to the polarization in contrast to the quantum wells, a polarization rate of $33 \%$ is measured. The optically pumped VCSEL is fabricated by metallic bonding, which allows the deposition of two dielectrics Bragg mirrors. The VCSEL with an active region based on InGaAs/InGaAsP quantum wells exhibits a lasing emission at 1.578 $\mu \mathrm{m}$ at room temperature under continuous wave operation. The VCSEL with an active region based on quantum wires shows a luminescence at $1.53 \mu \mathrm{m}$ strongly polarized along the direction [110] which is promising for the stabilization of in plane polarization of VCSEL emission.

Corresponding author: jean-michel.lamy@ens.insa-rennes.fr, Phone: + 33-2-23-23-84-64, Fax: + 33-2-23-23-86-18 


\section{Introduction}

VCSELs operating at $1.55 \mu \mathrm{m}$ represents big interest as laser source for optical telecommunication network. They present many advantages like their circular beam, spectral and spatial single-mode providing very efficient fibre coupling, their low-cost production give by the possibility to test each laser directly on the wafer. The main problem related with this VCSEL structures is the instability of the optical polarization [1] due to the circular symmetry design of its cavity. Different solutions have been carried out to control the polarization like using surface gratings [2], inserting birefringent material as one of the two Bragg mirrors [3, 4], etching elliptical mesa [5]. But these approaches need particular materials or increase the complexity of the device realization. Therefore, it would be preferable to utilize an active region based on anisotropic polarization gain to solve the problem of the polarization. VCSEL based on strained quantum-wells (QWs) grown on (311)A or (311)B oriented substrate evidences a strong polarized laser emission $[6,7]$. Another solution to get an active region with gain anisotropy without using a special substrate may be the QWires growth on InP(100). QWires structures have shown a polarization anisotropy of photoluminescence (PL) [8]. In contrast to the QWs these nanostructures present lot of advantages like lower threshold current, modulation at higher frequencies. In this letter, we present the growth and characterizations of InAs QWires on InP, the fabrication of a VCSEL device with an active region based on QWires.

\section{Growth and characterizations of the QWires}

InAs QWires have been grown on lattice matched $\mathrm{Ga}_{0.2} \mathrm{In}_{0.8} \mathrm{As}_{0.435} \mathrm{P}_{0.565}\left(\mathrm{Q}_{1.18}\right)$ alloy by gas source molecular beam epitaxy (GS-MBE), on (001) oriented InP substrate. After oxide removal and buffer growth, growth conditions have been carefully optimized in order to 
favour the spontaneous formation of QWires following the Stranski-Krastanov growth mode. Figure 1 is a $1 \times 1 \mu \mathrm{m}^{2}$ atomic force microscopy (AFM) image, realized in contact mode, of samples containing five layers of QWires, separated by a $30 \mathrm{~nm}$ thick $\mathrm{Q}_{1.18}$ spacer layer, the last layer being left uncovered for AFM measurement. QWires are clearly formed, and aligned along the $[1 \overline{1} 0]$ crystallographic axis. This preferential orientation mainly comes from an anisotropic stress relaxation, as already observed by several groups [9]. The surface organisation of QWires appears compact, and an important linear density of $30 \mathrm{~mm}^{-1}$ can be deduced. The structural dimensions of QWires, width, height and length have been measured to be respectively, $25 \mathrm{~nm}, 2-2.5 \mathrm{~nm}$ and $500-700 \mathrm{~nm}$. Figure 2 presents optical polarization dependent photoluminescence (PL) spectra for two different samples, the first one containing six layers of optimized QWires separated by a $30 \mathrm{~nm}$ spacer layer, and the second one seven layers of conventional InGaAs quantum wells (QWs), both being grown on $\operatorname{InP}(001)$ substrates. In order to measure the polarization rate of emitted light, a polarizer has been inserted between the sample and the input slit of the monochromator. The polarizer position $0^{\circ}$ corresponds to the situation when the polarizer axis is collinear to the [110] substrate orientation, and $90^{\circ}$ to $[1 \overline{1} 0]$. The intensities have been normalized on the intensity at $0^{\circ}$. The QWs sample exhibits a PL spectrum centred at $1.56 \mu \mathrm{m}$, and full width at half maximum (FWHM) close to $90 \mathrm{~nm}$. By changing the polarizer orientation, a slight intensity reduction is observed, as already reported for such InGaAs/InP hetero-interfaces [10]. Increasing this variation appears to be quite difficult, as the interface has to be controlled at the atomic scale. The QWires sample presents a PL spectrum also centred at $1.55 \mu \mathrm{m}$, and a larger $180 \mathrm{~nm}$ FWHM compared to QWs, related to the height dispersion. An important reduction of the intensity is observed when changing the polarizer orientation from [1 10$]$ to [110]. This clearly indicates that QWires emitted light presents a stronger polarization rate (PR), regarding the alignment of QWires on the substrate. 
Going further, Fig. 3 compares the integrated PL intensity (without any monochromator) performed on the same samples, depending on the orientation of an analyser inserted between the sample and the InGaAs photodiode. The reference angle $\left(0^{\circ}\right)$ has been fixed when the optical axis of the analyser is collinear to the [110] crystallographic direction. The PR is estimated as following: $P R=\frac{I[1 \overline{1} 0]-I[110]}{I[1 \overline{1} 0]+I[110]}$.QWs sample presents a rather homogenous variation, with a PR of $11 \%$. QWires sample exhibits important variations, with a clear minimum for an orientation perpendicular to the QWires ([110]), and a maximum of integrated PL intensity when the orientation is along the QWires ([1 $\overline{1} 0])$. These results reveal a PR of 33\% which is comparable with InAs QWires grown on InAlAs, evidence of the high quality of the growth [8]. This confrontation underlines the great interest in QWires compared to QWs for the realization of VCSEL structures.

\section{Micro-cavity realization}

Figure 4 is a schematic of fabrication process of VCSELs structure. In order to get a high reflectivity and a good thermal conductivity, dielectric materials have been preferred to epitaxial mirrors [11]. The dielectric materials used are amorphous silicon (a-Si) and amorphous silicon nitride $\left(\mathrm{a}-\mathrm{SiN}_{\mathrm{x}}\right)$ deposited using a magnetron sputtering system. A large 1.9 optical index contrast at $1.55 \mu \mathrm{m}$ wavelength enables the realization of efficient DBR mirrors achieving a $99.5 \%$ reflectivity with four and half periods. The $3.5 \lambda$-cavity active region consists in $\mathrm{InGaAs} / \mathrm{Q}_{1.18} \mathrm{QWs}$ or InAs/Q $\mathrm{Q}_{1.18}$ QWires on (001)InP realized by MBE and designed to optimize the absorption of the optical pump and maximize the stationary field overlap with the QWs or QWires (step 1). The lower Bragg mirror of 6 periods is deposited directly on the active region (step 2). An Au-In eutectic bonding is employed to transfer the 
sample on a silicon substrate (step 3,4,5). An epoxy resin is applied on the side of the component to protect the active zone while removing the InP substrate by mechanical polishing and chemical etching (step 6,7). The cavity is thus tuned in order to match the cavity mode with the active region PL emission. The process ends by the deposition of the second Bragg mirror constituted by 5 periods (step 8).

\section{QWs and QWires VCSELs characterizations}

The photopumping experiment consists in focusing a laser beam with a microscope objective on the front mirror of the VCSEL with a spot diameter of $10 \mu \mathrm{m}$. A CW YAG laser emitting at $1.064 \mu \mathrm{m}$ is used to pump optically the structure. The design of the VCSEL is determined by the size of the spot.

\section{Result}

Figure 5 shows the lasing spectra of the VCSEL with an active region based on $3 \times 3$ QWs $(3$ groups of 3 QWs located at each maximum field) with in inset the output power as a function of the density excitation pump power. The VCSEL showed a single mode spectrum at 1.578 $\mu \mathrm{m}$ under $\mathrm{CW}$ operation. The single mode laser emission is provide by the small size of the spot diameter of pump laser and the short cavity of $3.5 \lambda$. The laser emission has been obtained up to $35{ }^{\circ} \mathrm{C}$. As can be seen the lasing spectra at room temperature present a maximum output power of $380 \mu \mathrm{W}$ and a threshold power density of about $40 \mathrm{~kW} . \mathrm{cm}^{-2}$. A roll-over of the

output power curve can be seen for a pump density power higher than $45 \mathrm{~kW} . \mathrm{cm}^{-2}$. This phenomenon is occurred by the thermal heating of the microcavity. This thermal problem is well known for the VCSEL [12-14]. Because QWs exhibit a small PR as previously observed 
at the Fig. 3, the polarization of the laser emission is not stable and changes at any time and at any place on the sample, because of thermal and defect features. Figure 6 shows PL spectra of the VCSEL realized with an active region based on $3 \times 3$ layers of QWires. As can be seen two cavity modes are present at $1.36 \mu \mathrm{m}$ and $1.53 \mu \mathrm{m}$. Note that these QWires structures have recently demonstrated laser emission in edge emitting lasers containing three QWires layers, and they exhibit good properties at room temperature $\left(a 90 \mathrm{~A} / \mathrm{cm}^{2}\right.$ infinite threshold current per QWires layer, a $60 \mathrm{~K}$ characteristic temperature) (to be submitted). Nevertheless, QWires VCSEL does not present any laser emission, mainly because of a too weak modal gain. Two reasons can explain this low gain. First we have a size dispersion of the height of the QWires so a dispersion in the wavelength emission of the structure which induce a reduction of the gain for each wavelength. Second the height of the QWires is three times smaller than the thickness of the QWs, resulting in a lower overlap between the stationary field and the QWires, decreasing the effective modal gain. The inset compares the PL spectra of the parallel $[1 \overline{1} 0]$ and perpendicular [110] directions to the long axis of the QWires. The PL intensity in the direction [1 $1 \overline{1} 0]$ is more important than the PL intensity in the direction [110] and gives a PR of 33\% like for the structure of 6 QWires layers. That shows the conservation of the QWires properties in the microcavity and it is promising to get the control on the polarization of the laser emission.

\section{Conclusion}

We have grown and studied optically InAs QWires on $\operatorname{InGaAsP} / \operatorname{InP}(100)$. They are well organized on surface and aligned in the direction [1 $\overline{1} 0]$. They exhibit a peak of PL at 1.55 $\mu \mathrm{m}$ with strong polarization dependence. Using QWires, an increase of the PR has been 
obtained compared to QWs (33 and $11 \%$ respectively in the direction [1 10$]$ ). These properties of QWires are preserved in the microcavity. The modal gain provided by the $3 \times 3$ layers of QWires is too weak to reach the laser emission. Further optimizations are needed, like increasing gain by stacking more layers, and reducing spacers thickness. All these results should be promising for the realization of a VCSEL with a stabilized polarization.

Acknowledgements T. Rohel and F. Thoumyre are greatly acknowledged for their technical help in growth by MBE and fabrication process of the VCSEL. 


\section{References}

[1] J. Martin-Regalado, F. Prati, M. San Miguel, and N. B. Abraham, IEEE J. Quantum Electron. 33, 765 (1997).

[2] P. Debernardi, J. M. Ostermann, M. Feneberg, C. Jalics, and R. Michalzik, IEEE J. Sel. Top. Quantum Electron. 11, 107 (2005).

[3] T. Mukaihara, N. Ohnoki, Y. Hayashi, N. Hatori, and F. Koyama, IEEE J. Sel. Top. Quantum Electron. 1, 667 (1995).

[4] M. S. Park, B. T. Ahn, B. S. Yoo, H. Y. Chu, H. H. Park, and C. J. Chang-Hasnain, Appl. Phys. Lett. 76, 813 (2000).

[5] H. J. Unold, M. C. Riedl, R. Michalzik, and K. J. Ebeling, Electron. Lett. 38, 77 (2002).

[6] Y. L. Okuno, J. Geske, K. G. Gan, Y. J. Chiu, S. P. DenBaars, and J. E. Bowers, Appl. Phys. Lett. 82, 2377 (2003).

[7] H. Uenohara, K. Tateno, T. Kagawa, Y. Ohiso, H. Tsuda, T. Kurokawa, and C. Amano, IEEE J. Sel. Top. Quantum Electron. 5, 537 (1999).

[8] L. González, J. M. García, R. García, F. Briones, J. Martínez-Pastor, and C. Ballesteros, Appl. Phys. Lett. 76, 1104 (2000).

[9] J. M. Garcia, L. Gonzalez, M. U. Gonzalez, J. P. Silveira, Y. Gonzalez, and F. Briones, J. Cryst. Growth 227/228, 975 (2001).

[10] S. Cortez, O. Krebs, and P. Voisin, J. Vac. Sci. Technol. B 18, 2232 (2000).

[11] C. Levallois, A. Le Corre, S. Loualiche, O. Dehaese, H. Folliot, C. Paranthoen, F. 
Thoumyre, and C. Labbe,

J. Appl. Phys. 98, 43107 (2005).

[12] A. Karim, S. Björlin, J. Piprek, and J. E. Bowers, IEEE J. Sel. Top. Quantum Electron. 6, 1244 (2000).

[13] J. Piprek, H. Wenzel, H.-J. Wünsche, D. Braun, and F. Henneberger, Proc. SPIE 2399, 605 (1995).

[14] M. Guden and J. Piprek, Model. Simul. Mater. Sci. Eng. 4, 349 (1996). 


\section{Figures captions}

Fig. 1 (online colour at: www.pss-a.com) AFM image from the last layer of five layers of InAs QWires on $\operatorname{InP}(001)$ growth by MBE.

Fig. 2 (online colour at: www.pss-a.com) Polarization dependence PL spectra of QWire structures compared with QWs at room temperature.

Fig. 3 (online colour at: www.pss-a.com) Integrated PL intensity of 7 QWs and 6 QWires layers as function of the direction. The intensities are respectively normalized on the intensity on the direction $[1 \overline{1} 0]$ for the QWs an QWires.

Fig. 4 (online colour at: www.pss-a.com) Realization of the microcavity.

Fig. 5 (online colour at: www.pss-a.com) Laser emission of the $3 \times 3$ QWs VCSEL at 1.578 $\mu \mathrm{m}$ with in inset the output power as a function of the density pump power.

Fig. 6 (online colour at: www.pss-a.com) PL spectra of the $3 \times 3$ QWires layers VCSEL. 
FIG1

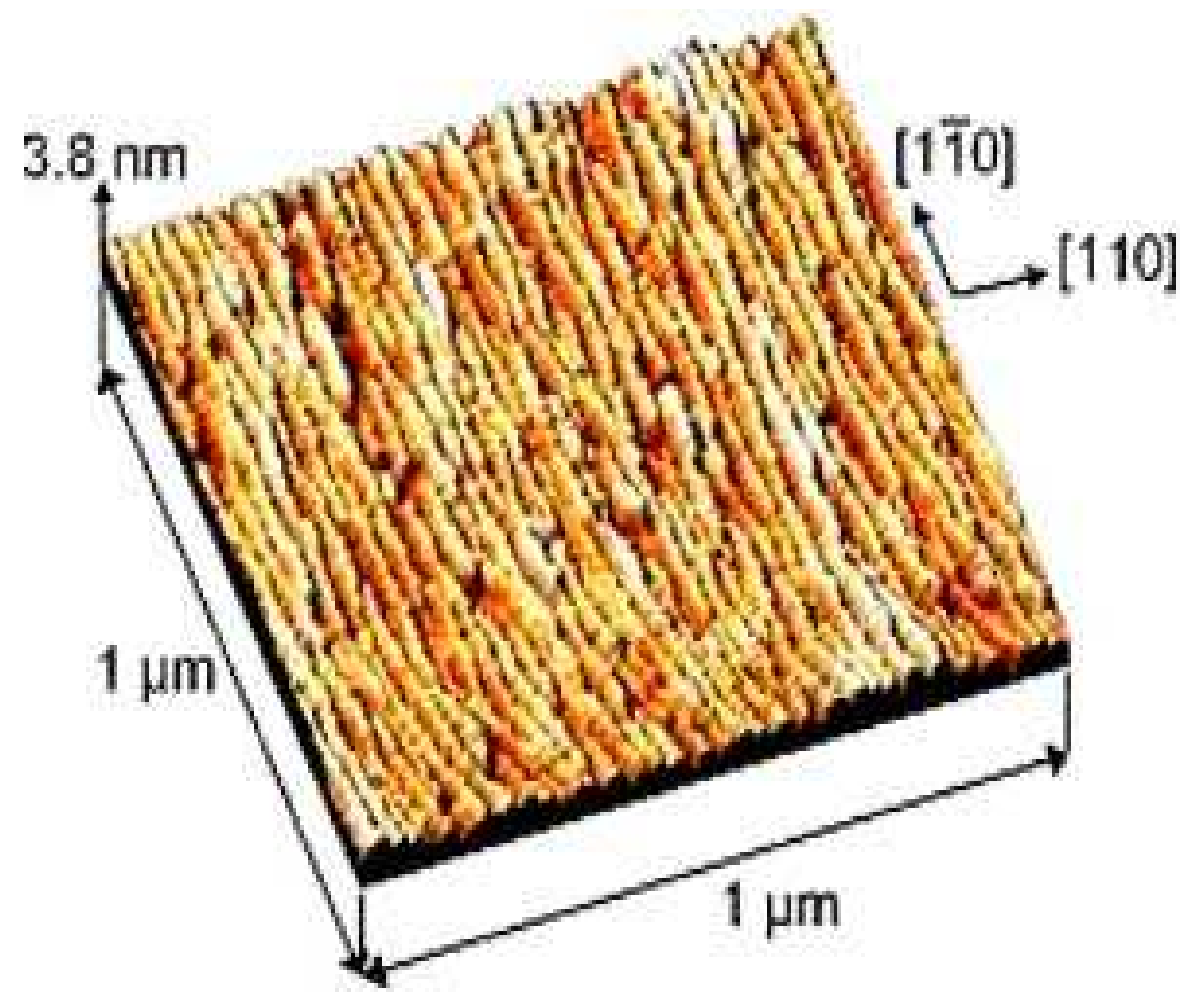


FIG2

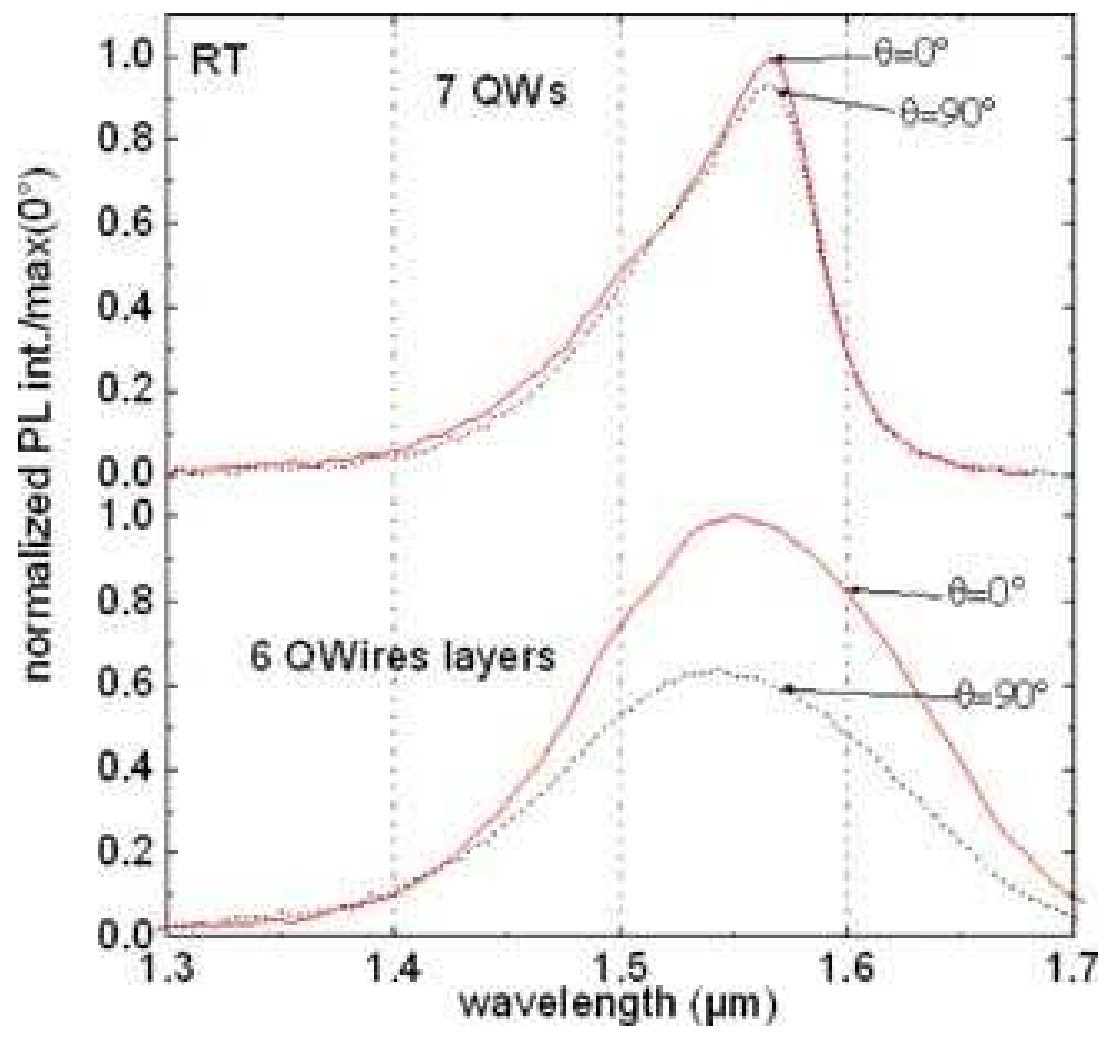


FIG3

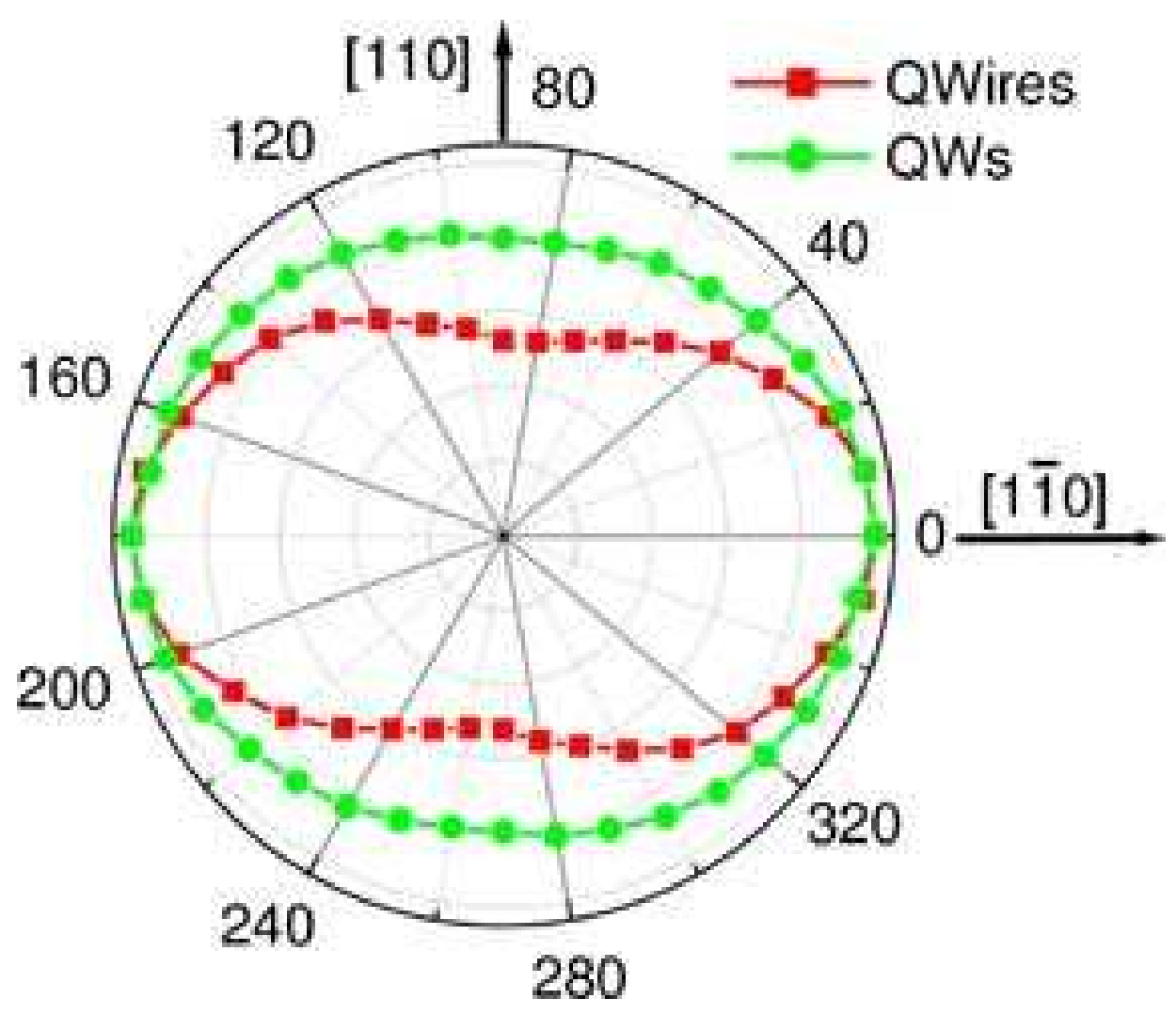




\section{FIG4}

(1)

(2)

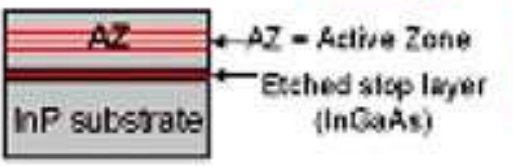

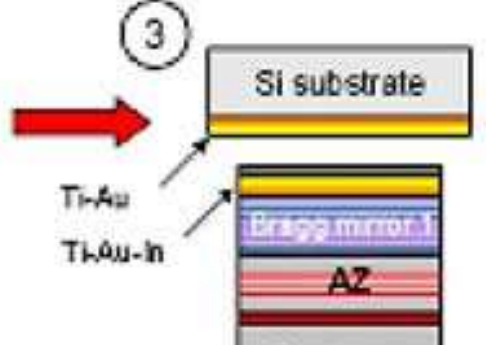

hP substate
(8)

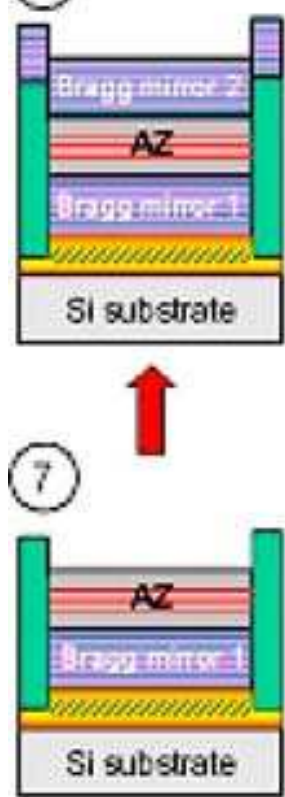

1. Actue zone epitaxy

2. Depositon of the frst Eragg minor

3. Metalization of the substrates

4. Fomation of the Auln aloy

5. Costing of the sample edges with epoxy resn

6. Mechanical ninring

7. Complete withdrawal of the sutsirate $\alpha \operatorname{InP}$ and the stop etch layer by wel process

8. Depceiton of the and Eragg mirror

(6)

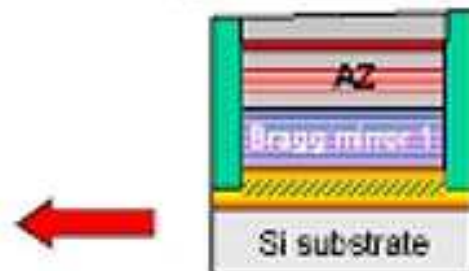

(4)

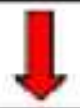

Si substrate

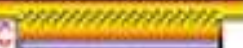

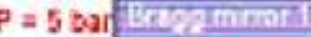

$A 2$

subsrate

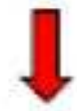

(5) InP substrate

A2

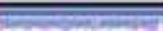

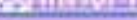

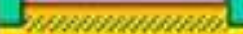

Si subsrate 


\section{FIG5}

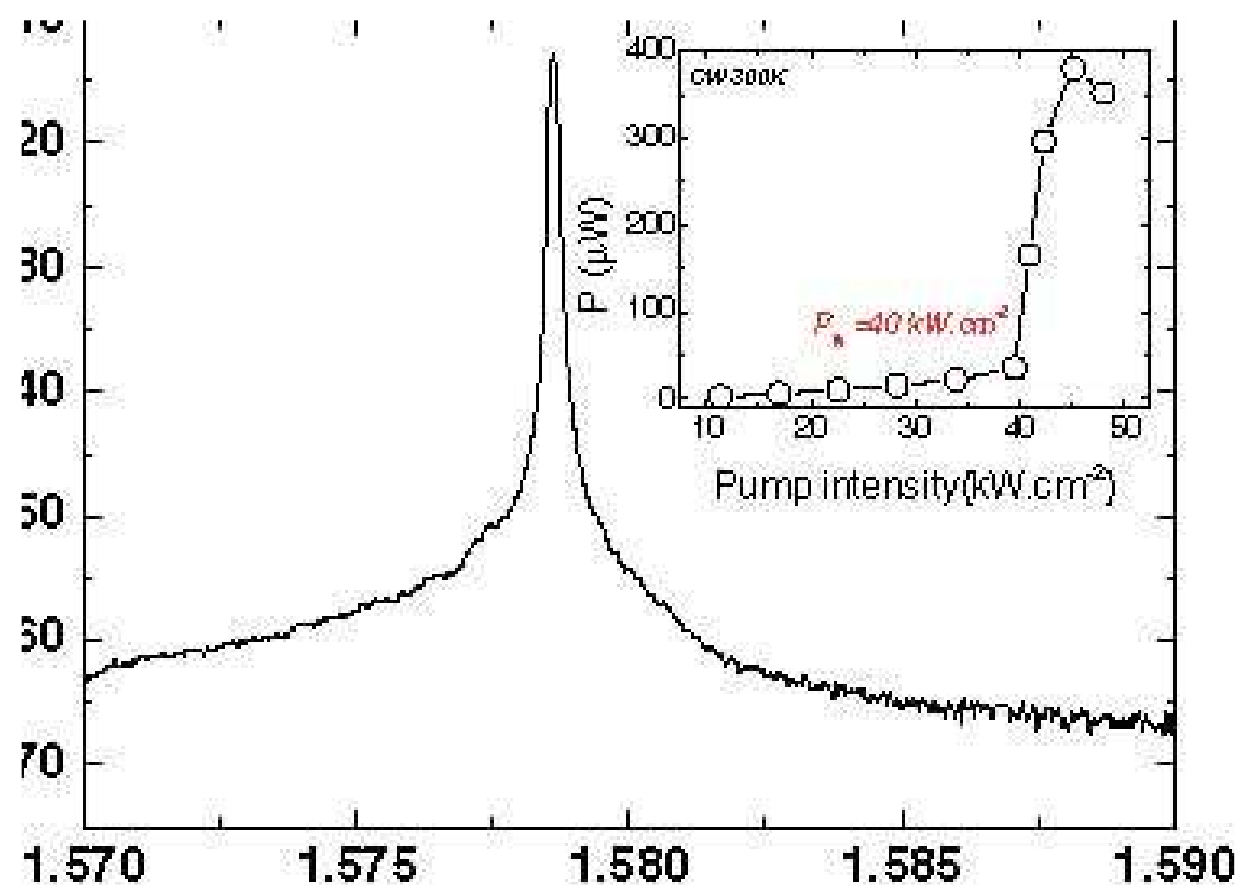


FIG7

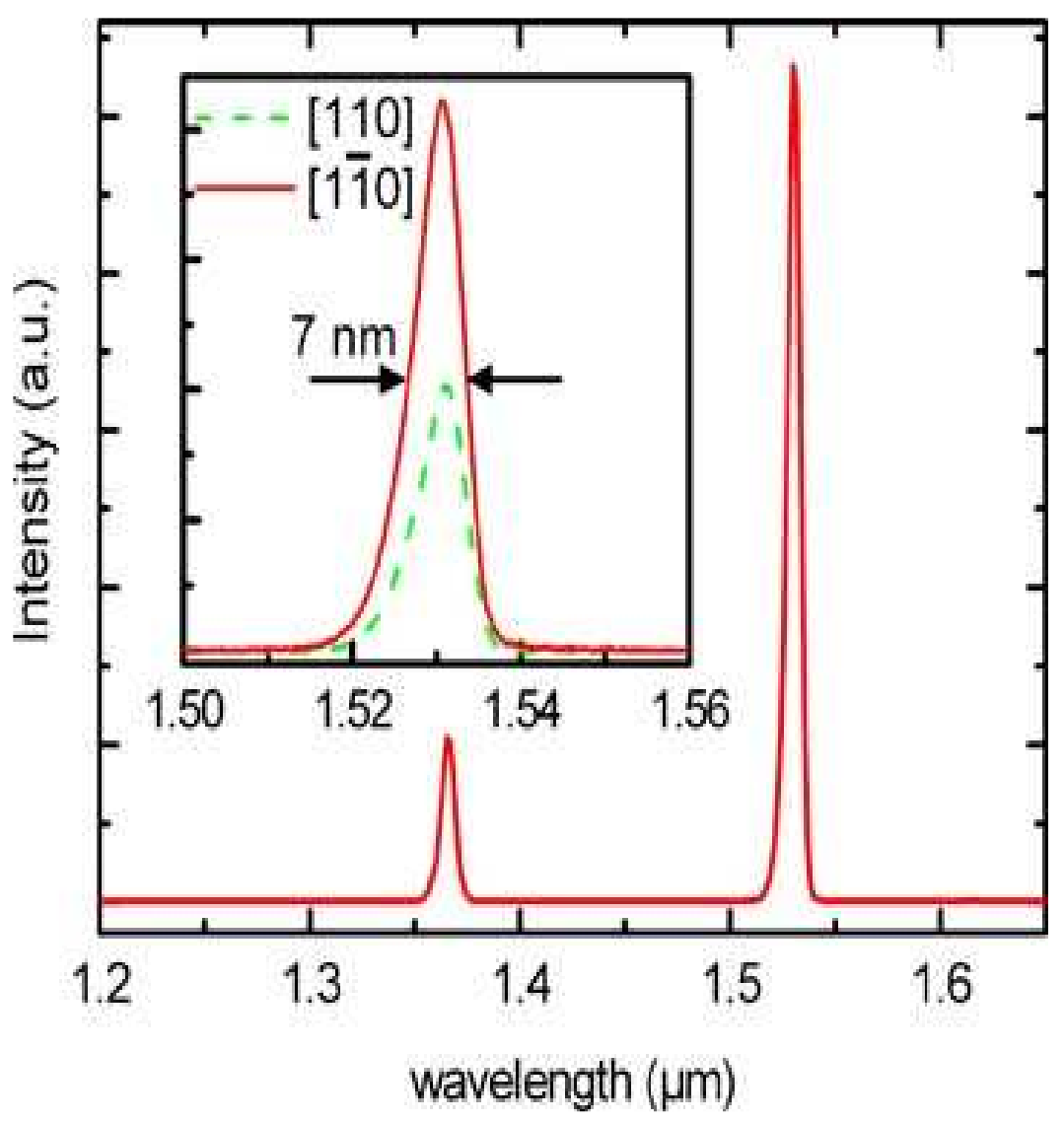

